\title{
Conflits et justice au Moyen Âge. Normes, loi et résolution des conflits en Anjou aux $\mathrm{XI}^{\mathrm{e}}$ et $\mathrm{XII}^{\mathrm{e}}$ siècles
}

\section{Bruno Lemesle}

\section{(2) OpenEdition Journals}

Édition électronique

URL : https://journals.openedition.org/cem/11236

DOI : $10.4000 /$ cem. 11236

ISSN : 1954-3093

Éditeur

Centre d'études médiévales Saint-Germain d'Auxerre

Édition imprimée

Date de publication : 15 août 2009

Pagination : 427-433

ISSN : 1623-5770

\section{Référence électronique}

Bruno Lemesle, "Conflits et justice au Moyen Âge. Normes, loi et résolution des conflits en Anjou aux XIe et XIIe siècles », Bulletin du centre d'études médiévales d'Auxerre / BUCEMA [En ligne], 13 | 2009, mis en ligne le 15 septembre 2009, consulté le 22 septembre 2022. URL : http://journals.openedition.org/ cem/11236; DOI : https://doi.org/10.4000/cem.11236

Ce document a été généré automatiquement le 22 septembre 2022.

\section{c) (i) (2)}

Creative Commons - Attribution - Pas d'Utilisation Commerciale - Partage dans les Mêmes Conditions 4.0 International - CC BY-NC-SA 4.0

https://creativecommons.org/licenses/by-nc-sa/4.0/ 


\title{
Conflits et justice au Moyen Âge. Normes, loi et résolution des conflits en Anjou aux $\mathrm{XI}^{\mathrm{e}}$ et $\mathrm{XII}^{\mathrm{e}}$ siècles
}

\author{
Bruno Lemesle
}

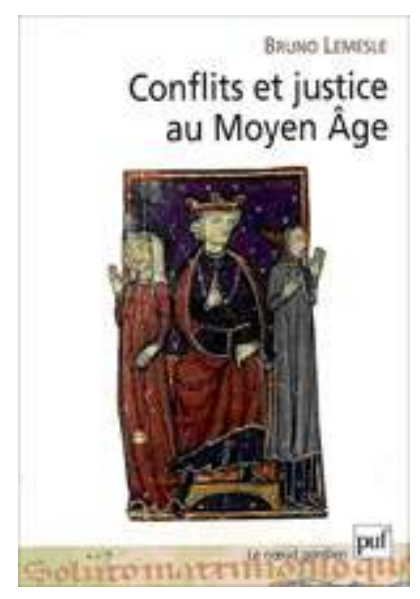

1 Pourquoi étudier les conflits et plus spécialement ceux qui relèvent des cours judiciaires ? Ce choix est guidé par le fait que ces conflits sont des révélateurs des relations de pouvoir dans lesquelles des communautés sont prises; si, en effet, les parties en litige sont le plus souvent des individus, ils agissent rarement seuls. Ce sont les tensions multiples au sein de la société et entre des pouvoirs rivaux qui sont ainsi révélées avec les enjeux pour les parties impliquées. Les prendre comme objet d'étude conduit donc à aborder à peu près tous les champs de l'histoire sans cloisonnement, aussi bien le domaine politique que religieux, social qu'économique, etc.

2 Un autre intérêt à l'étude des conflits portés en justice est que les procédures et les modes de résolution sont communs, à cette époque, aux diverses causes et aux 
différentes juridictions et que l'évolution qui se perçoit au XII siècle, même si elle amène à nuancer cette affirmation, ne la contredit pas radicalement ; certes, les causes traitées dans les cours ecclésiastiques présentent des particularités mais ce qui est commun à toutes dans la manière de les résoudre l'emporte sur les différences. Les procédures constituent aussi par elles-mêmes un des objets de ce livre car leur mutation au cours de ces deux siècles est un révélateur des transformations dans les relations de pouvoir.

3 L'époque est une période de transformations : on passe d'une société formée de petites communautés locales, où le pouvoir du prince, le comte d'Anjou, doit tenir compte de multiples pouvoirs locaux, qui sont des pouvoirs seigneuriaux, à une société beaucoup plus fermement dominée par le prince et où l'autorité de l'Église sur la société s'accroît également. Or la justice reflète bien ces transformations. Enfin, la période est très riche en documents dans cette région, ce qui m'a permis de concentrer l'étude en repérant des évolutions significatives, en mettant en valeur à la fois des données sérielles et en constituant des dossiers étoffés, chose paradoxalement plus difficile à réaliser pour le XIII ${ }^{\mathrm{e}}$ siècle.

Les documents sont les chroniques, celles des comtes d'Anjou, rédigées à plusieurs moments entre la fin du XI ${ }^{e}$ siècle et la seconde moitié du XII ${ }^{e}$ siècle. Des annales, écrites dans les scriptoria monastiques. Mais, surtout, le matériau est constitué par les chartes et notices très abondamment produites dans cette région entre le milieu du $\mathrm{XI}^{\mathrm{e}}$ siècle et le milieu du $\mathrm{XII}^{\mathrm{e}}$ siècle. La plupart émanent des établissements religieux et une part plus faible est constituée de diplômes comtaux et royaux. L'intérêt de ces actes de la pratique est qu'ils présentent des caractères de narrativité, ce qui est un des traits de ces documents des régions de la Loire. Ils contiennent donc souvent des récits circonstanciés ; or, précisément, ceux qui relatent des conflits en font partie au point qu'on peut dire que les actes les plus narratifs sont souvent ceux qui exposent des conflits.

Depuis le milieu des années 1980, l'anthropologie historique a transformé la manière de faire l'histoire de la justice et des conflits à cette époque. On peut dire que cette période centrale du Moyen Âge a été privilégiée puisque les deux introducteurs de l'anthropologie des conflits en France sont Patrick Geary et Dominique Barthélemy, dans la mesure où ils l'ont d'abord fait à partir d'études qui privilégiaient le $\mathrm{XI}^{\mathrm{e}}$ siècle. Pour ma part, dans ce livre, j'ai clairement assumé l'héritage anthropologique. Dans l'introduction, j'ai rappelé quels en ont été les apports. Mais j'ai aussi indiqué ses limites. L'anthropologie juridique est un modèle qui a sa propre histoire - qui bien entendu a beaucoup plus de vingt ans même si ce n'est que relativement récemment que l'on parle de "règlement des conflits », et il est essentiel de la faire afin d'en comprendre les présupposés.

6 Après un chapitre intitulé " prémisses » consacré à l'examen critique des sources, le deuxième chapitre du livre intitulé "Les assemblées de justice " analyse les litiges réglés devant des cours. Si l'objectif est de comprendre comment les hommes de cette époque réglaient leurs conflits et quelles stratégies ils mettaient en œuvre, il importe au préalable de définir les cadres dans lesquels ces stratégies s'effectuaient. C'est dans cette perspective que les cours, l'autorité dont elles relèvent et les enjeux qui y sont débattus sont étudiés. Classiquement, les historiens ont évalué les cours féodales en les comparant aux plaids carolingiens, mais l'idéalisation des seconds a eu pour pendant le noircissement des premières. 
La justice féodale aurait été de moins en moins publique, ce que la composition des cours et l'entourage des autorités auraient rendu manifeste. Les cours comtales auraient perdu en audience tandis que les cours à caractère privé auraient enserré les hommes en prenant en partie la place des premières. Les attributs de l'ancienne justice qui demeuraient étaient tenus pour des imitations ou pour des aspects dégradés.

7 Après avoir fait l'histoire de ces appréciations, j'ai essayé de montrer que les auteurs contemporains évoquaient rarement les assemblées judiciaires dans les mêmes termes que l'historiographie. En réalité, le clivage entre une justice publique carolingienne et une justice féodale aux caractères privés et dégradés obscurcit sa compréhension. Mieux valait partir de la manière dont les contemporains la percevaient, observer ce qu'ils en disaient et montrer l'extrême importance qu'ils accordaient à la publicisation des décisions, qui n'est pas le fait des seules assemblées judiciaires.

8 Les autres clivages généralement mis en avant sont moins nets qu'on ne l'a dit. Par exemple, les relations entre la justice du comte et celle de l'évêque. Ce dernier aurait profité de l'affaiblissement de la puissance comtale dans le dernier tiers du $\mathrm{XI}^{\mathrm{e}}$ siècle pour imposer son autorité et certaines décisions. Au XII ${ }^{\mathrm{e}}$ siècle, en revanche, le raffermissement du pouvoir princier aurait été générateur de tensions durables et de fortes rivalités en matière judiciaire entre ces deux autorités. Mais, si les tensions et rivalités ont été réelles, les connivences l'emportaient largement, au XI ${ }^{e}$ siècle surtout, mais encore au XII ${ }^{e}$ siècle. De même, les clivages entre justice châtelaine laïque et justices ecclésiastiques sont moins sensibles qu'on ne l'a dit, du fait de l'absence d'étanchéité des justices jusque dans la première moitié du XII ${ }^{\mathrm{e}}$ siècle.

9 Il n'existe pas encore d'institution en Anjou au XII ${ }^{e}$ siècle, hormis l'Église. Pourtant, de premiers jalons menant à une administration ont été posés. Le personnel comtal s'étoffe dès la fin du $\mathrm{XI}^{\mathrm{e}}$ siècle ; avec le principe de la délégation de la justice au sénéchal dans la seconde moitié du XII ${ }^{\mathrm{e}}$ siècle, il devient difficile d'échapper à la justice du comte même si certains seigneurs croient pouvoir le faire. Pour autant, je ne constate pas de tentative de réduire les justices seigneuriales. Les rivalités ne sont pas forcément là où on les attend; par exemple, on en repère dans les conflits d'influence entre les justices monastiques et celle de l'évêque. Quant aux justices comtale et épiscopale, si elles sont le plus souvent en situation conflictuelle dans la première moitié du XII ${ }^{e}$ siècle, les connivences ne disparaissent pas et le choix des évêques d'Angers par le roi Henri II contribue sérieusement à limiter les tensions.

10 L'étude des normes occupe une place importante. Pourtant les références aux normes sont numériquement peu élevées dans les documents et elles sont plus souvent implicites qu'explicites. J'ai mené leur examen à travers diverses approches en essayant de dépasser la dichotomie traditionnelle qui oppose le droit à ses applications pratiques. Il s'agissait de prendre en considération les usages que les acteurs font des normes en voyant comment ils les manipulent, ou tentent de les manipuler à leur avantage, d'une part, mais aussi d'observer leur formation.

11 L'étude des conflits offre l'un des meilleurs points de vue pour les appréhender. Les litiges naissent fréquemment des dons et des ventes aux établissements religieux, mais, s'ils sont liés à la circulation des biens, c'est parce que leurs 
bénéficiaires les immobilisent le plus souvent dans le patrimoine monastique. La litigiosité de cette époque est un indicateur des transformations structurelles et économiques. L'essor économique rend les établissements ecclésiastiques aptes à gérer eux-mêmes leurs biens, en dépit de difficultés ponctuelles. La croissance démographique leur donne des moyens. La plupart des conflits peuvent être rapportés au dynamisme économique des moines et à la volonté de gestion rationnelle qui l'accompagne. Ainsi s'explique la tendance à évincer les petits aristocrates et les personnes de moindre envergure sociale exploitant les terres en usufruit, au profit d'exploitants dépendant directement des détenteurs de la terre. Cette rationalité exigeait un droit moderne qui ne reconnaissait pas les normes sociales où l'aliénation des biens était rarement absolue. Naturellement, les transactions tempèrent parfois substantiellement ce que disent les notices. Leur rationalité affichée s'accompagne des justifications spirituelles aux dons avec des promesses eschatologiques aux donateurs: le sens religieux rend possible l'acte économique; l'un et l'autre se conjoignent.

Les litiges permettent également de fixer des normes, de préciser des droits, de les inscrire dans l'espace, d'établir des règles. Les conventions sont liées à cela et non aux structures d'encadrement supposées plus ou moins faibles de la seigneurie, voire de l'autorité comtale. Elles constituent des enjeux de négociations entre des pouvoirs, des institutions (ecclésiastiques) et des communautés d'habitants et de dépendants. Il y a là un enjeu considérable puisqu'elles fournissent des socles de légitimité et qu'elles offrent des référents lorsque les conflits surgissent.

13 Avec le droit canonique, nous sommes apparemment sur un terrain différent. Pourtant, lorsque l'on observe les mentions, on constate que, en dépit de leur nombre plus élevé par rapport aux références coutumières, le total demeure médiocre. Les rédacteurs de notices sont plus enclins à l'allusion qu'à la citation précise des sources d'autorité. Même dans des notices à tonalité juridique, leurs rédacteurs ne mettent pas fortement l'accent sur le droit canonique; face à des compétiteurs laïcs, les moines préfèrent défendre le droit de leur église en fondant leur action sur des principes traditionnels plus facilement admis par la société.

$14 \mathrm{Au} \mathrm{XII}$ siècle cependant, le droit savant pénètre peu à peu les résolutions des litiges ecclésiastiques. Mais, là encore, il importe de mettre un bémol car le droit canonique et le droit romain demeurent souvent confinés à des techniques de réfutation sans que soient introduites de réelles mutations de procédure. Et l'on voit que les actions rituelles ne connaissent aucun recul de leur fonction probatoire. Les preuves coutumières ne sont pas utilisées à la place des preuves fondées sur le droit savant car elles ne leur sont nullement antithétiques : elles sont utilisées conjointement, comme s'additionnant les unes aux autres, sans s'exclure.

15 La mutation que j'ai analysée se déroule au long du XII ${ }^{e}$ siècle; c'est à travers l'étude des preuves et des épreuves qu'elle est menée de la façon la plus adéquate car elle permet de définir la transition progressive, assez lente, entre des plaids fondés sur une relation de type agonistique (où un défi est lancé) et des plaids dans lesquels ce principe recule. Mutation qui n'est pas facile à repérer puisque les cadres procéduraux n'évoluent guère, en dehors des cours ecclésiastiques, et que les preuves utilisées demeurent peu ou prou identiques. 
16 Au plan historiographique, j'ai évoqué à grands traits la formation du paradigme ancien fondé sur une conception évolutionniste de la justice, une conception qui a eu la vie dure en dépit des écrits de Louis Gernet qui mettait en garde contre elle. Ainsi, les épreuves surnaturelles auraient été remplacées par les preuves rationnelles, les vengeances auraient évolué vers les duels judiciaires, les témoins de solidarité (les cojureurs) auraient laissé place aux témoins de connaissance. Pourtant, rien ne se passe tout à fait comme cela : les duels persistent au-delà du $\mathrm{XII}^{\mathrm{e}}$ siècle en dépit de l'émergence des procédures savantes; même après le $4^{\mathrm{e}}$ concile de Latran de 1215 , les ordalies ne disparaissent pas totalement et leur mémoire se perpétue plusieurs siècles après. Les duels sont contemporains des vengeances et ne sont pas leur substitut ritualisé. Quant aux témoins de connaissance, ils existent déjà dans les périodes anciennes ainsi qu'au haut Moyen Âge.

17 La relative abondance de la documentation angevine a permis de faire un bilan comparé des pratiques des ordalies et des duels. On y confirme que les ordalies ne sont pas, contrairement à une idée tenace, le moyen le plus courant de régler les litiges; leur utilisation est restée marginale. Mais au terme de cette comparaison, j'ai surtout découvert que près de la moitié des ordalies proposées étaient mises en œuvre alors que seule une proposition de duel judiciaire sur cinq connaissait un début d'effectuation. Ce trait souligne le caractère dissuasif des duels judiciaires, qui, davantage que les ordalies, participent de relations de type agonistique entre les participants au plaid. L'autre différence notable est que les ordalies circonstanciées disparaissent des actes de la pratique après 1130 alors que les duels se poursuivent. Cette mutation trouve son explication dans l'attitude des autorités ecclésiastiques, qui en est la cause, et dans la réticence des laïcs qui manifestaient parfois une défiance envers les ordalies et leur préférence pour les duels.

18 Le recul des épreuves vers les années $1120-1130$ a pour corollaire un mode différent de recours aux témoins. Yves de Chartres sait définir les témoins de connaissance, mais il n'oppose pas ces témoins de vérité aux témoins de solidarité. Déjà au $\mathrm{XI}^{\mathrm{e}}$ siècle, on voit que les contemporains n'accordent pas la même foi aux témoins partisans et aux témoins supposés être neutres : le témoignage impartial a davantage de valeur. Naturellement ce n'est pas parce qu'un auteur évoque l'impartialité des témoins que nous devons le croire, mais l'on voit bien que l'on touche ici aux manières de penser des contemporains, ce qui nous importe, et que ce constat prend à contre-pied les thèses généralement admises.

$19 \mathrm{Au} \mathrm{XII}$ siècle, la nouveauté principale est que les témoins, à la cour du comte ou à celle de l'évêque, peuvent être entendus, et que leur témoignage, écouté sous la foi $\mathrm{du}$ serment, suffit aux juges, alors que les serments, dans les relations agonistiques, sont le prélude aux épreuves surnaturelles. Mais, au XI comme au XII ${ }^{\mathrm{e}}$ siècle, les preuves ne fonctionnent pas comme aujourd'hui et, s'il faut employer le terme sans hésitation, à l'instar des contemporains, il importait tout autant d'établir comment ils les concevaient et quel était leur régime. Au même titre que les argumentations, que l'on peut ranger à leur côté, elles permettaient de justifier les décisions de ceux qui jugeaient ; cependant bien des affaires nous révèlent qu'elles n'étaient pas toujours déterminantes et que les rapports de forces et les alliances pouvaient être bien plus décisifs. 
20 La difficulté majeure à laquelle l'historien est confronté tient à ce que sa documentation pour un tel sujet est constituée de récits construits. Cela induit un formalisme de genre auquel les auteurs conforment les faits rapportés. La réitération des arguments, des allégations, des modes de preuves ou des récits de procédures reflète les conventions, indique quelles étaient les stratégies les meilleures, les normes acceptées, bref fournissent un bon indicateur de l'horizon des valeurs communes. Mais je n'ai pas voulu faire seulement l'histoire du système d'interprétation des auteurs médiévaux. Il fallait aussi, autant que possible, découvrir ce que font les acteurs, et pas uniquement ce qu'ils disent, prétendent, cherchent à avoir ou à faire.

21 Les notices s'avèrent souvent être des armes de combat; qu'elles servent à préparer des litiges futurs ou qu'elles inscrivent la mémoire de l'établissement religieux, les énoncés révèlent toujours une grande maîtrise de l'exposé de la cause, des griefs, des arguments. Mais on y voit que si l'exposé narratif est bien celui du déroulement des plaids, les auteurs se livrent à une réécriture avec rectifications, omissions, voire inventions.

22 J'ai dit ma dette à l'égard des travaux de l'anthropologie tout en ajoutant que je ne pouvais pas m'en satisfaire. L'anthropologie a incontestablement transformé une pensée dans le champ des conflits, entre autres par la prise en considération des litiges réglés hors des cours, par le refus de dresser une barrière entre ce qui s'y passe quand on y rend la justice et tout ce qui se règle en dehors, ou en tenant le choix d'une cour pour une composante du règlement. Pourtant, l'idée que la recherche de la résolution et de la paix serait inhérente aux conflits est un postulat qu'il convenait de corriger. Après tout, la plupart des documents qui sont parvenus jusqu'à nous n'existent que parce qu'ils exposent le contenu d'une résolution. L'accent mis par l'anthropologie juridique sur les négociations, les compromis et les transactions a contribué à montrer des plaids pacificateurs. Il arrive pourtant que, si les plaids ramènent la paix, ils contribuent aussi à justifier et à légaliser des avantages obtenus par la violence, en particulier au $\mathrm{XI}^{\mathrm{e}}$ siècle.

Les litiges sont partout dès lors que des pouvoirs et des autorités sont en situation de rivalité, dès lors que des biens, des objets et des signes de prestige sont en jeu. Étudier les conflits sous toutes leurs facettes exigeait de ne pas privilégier la seule étude de leur résolution. Ces multiples petits conflits devaient être analysés au travers des acteurs et des enjeux parce qu'ils révèlent les dynamiques d'une société et ce qui agit sur elle. En même temps cette multiplicité ne devait pas faire perdre de vue les lignes de force qui sont dans les moyens par lesquels la justice du comte s'impose au XII siècle et dans l'extension des compétences ecclésiastiques en matière de causes. 
INDEX

Mots-clés : conflit, justice, norme

Index géographique : France/Anjou 